\title{
Landschaftsbeobachtung - ein Beitrag zur integrierten, langfristigen Umweltbeobachtung in der Schweiz
}

\author{
Als sie an den See kamen, sagte Springende Maus: \\ "Beschreib mir den Ort." \\ Und der Wolf versuchte es. Er beschrieb, daß die Berge \\ riesig waren und purpurne Spitzen hatten und daß sie aus \\ dem Nebel herausragten und daß alles still und friedlich \\ war: \\ "Ich könnte eine Ewigkeit damit zubringen, dir alle Ein- \\ zelheiten zu beschreiben", sagte der Wolf. "Aber die Ein- \\ zelheiten für sich genommen sind ganz unbedeutend."
}

Brian Patten

in "Springende Maus - Ein indianisches Märchen»

\section{Landschaft als Gegenstand von Beobachtung}

\section{Vorspann}

Seit 1986 arbeitet die Schweiz. Kommission für Umweltbeobachtung (SKUB), eingesetzt von der Schweiz. Akademie für Naturwissenschaften und finanziell unterstützt vom BUWAL, an einem Konzept für die Einführung eines Umweltbeobachtungssystems in der Schweiz. Vor kurzem erschien ein ausführlicher Bericht mit dem Vorschlag, eine integrierte ökosystembezogene Umweltbeobachtung in der Schweiz einzuführen. In diesen Bericht flossen die Resultate aus den Forschungsarbeiten der interdisziplinären SKUB-Arbeitsgruppe ein. Auf der Grundlage der vorgeschlagenen Basisprogramme versucht seit 1993 das LEKUB (Lenkungs- und Koordinationsorgan Umweltbeobachtung, koordiniert vom BUWAL), eine koordinierte Umweltbeobachtung zu realisieren.

\section{Umweltvorsorge bedingt Umweltbeobachtung}

Neben der Erkenntnis, daß unsere Umwelt ja eigentlich unsere Mit-Welt ist und ihre Gesundheit auch unser Dasein beeinflußt, sowie dem immer häufiger postulierten «Recht der Natur» führen uns auch juristische Gegebenheiten dazu, vermehrt und mit einem sensibilisierten Bewußtsein die Umwelt zu beobachten. Das eidgenössische Umweltschutzrecht verpflichtet den Bund und die Kantone, «Erhebungen über die Umweltbelastung» durchzuführen und den Erfolg der Maßnahmen zu überprüfen, die nach dem Umweltschutzgesetz getroffen werden (Art. 44 USG - SR 814.01).

In der Schweiz wird eine langfristige Umweltbeobachtung nur ansatzweise und in der Regel sektoriell betrie- ben (ZIMMERMANN 1900, SCHEURER 1991). Ein integraler Ansatz wird bis anhin vermißt. Die bestehenden Ansätze zur Umweltbeobachtung leiden einerseits unter der Aufgabenteilung zwischen Bund und Kantonen. Die Verknüpfung regional und lokal gewonnener Datensätze mit gesamtschweizerischen Daten ist erschwert, da entsprechend den Besonderheiten der jeweiligen Vollzugspolitik mitunter ganz verschiedene Parameter, Meßmethoden und Meßperioden verwendet werden. Neben diesen «vertikalen Integrationsproblemen» stellt andererseits die sektorielle Beobachtungskonzeption mit ihren unkoordiniert nebeneinander gestellten Meßnetzen auch eine «Vielzahl von Problemen für eine horizontale Integration" (KNOEPFEL 1992: 3) dar. Bedingungen zu einer integralen Umweltbeobachtung sind nicht erfüllt. Dies führt nicht nur zu ungenügenden Beobachtungsdaten, sondern auch zu Schwierigkeiten bei der Umweltberichterstattung und somit zum mangelnden Gesetzesvollzug.

\section{Integrierte Umweltbeobachtung bedingt auch Landschaftsbeobachtung}

Das ehrgeizige Projekt einer integrierten Umweltbeobachtung verlangt mehr als einen einseitigen ökologieorientierten Ansatz. "Die Umwelt des Menschen ist nicht das, was man sieht, die Umwelt ist sozial», schreibt der Architektursoziologe Lucius BURCKHARDT (1985: 390), und er deutet damit die Vielschichtigkeit des Begriffs «Umwelt» an. Der Mensch und die ihn «umgebende Natur» (FLACH 1986: 17) stehen untrennbar zueinander in Beziehung. Wenn Hanspeter PADRUTT (1984: 33) den Weg von der Um-Welt, "die wir besitzen und ausbeuten", hin zur Welt, «in der wir wohnen», vorschlägt und wenn Bernhard WALDENFELS (1986: 37) von der Zwischenwelt, einer Landschaft als sozialem Lebensraum spricht, so wird in diesen Äußerungen die Verbundenheit von Organismus und Welt deutlich. Wir sind genötigt, die Landschaft als Mensch-Natur-Beziehung zu verstehen. In diesem Sinn spielt die Landschaft «als Handlungs- und Anschauungsraum» (WALDENFELS 1986) in ihrem Wesen für das Umweltverständnis eine wesentliche Rolle. Die Absicht, Umwelt integral zu beobachten, bedingt somit auch die Beobachtung der Landschaft. Mehr noch: Die Umweltbeobachtung muß sich unmißverständlich am holistischen Charakter der Landschaft orientieren.

Peter Glauser, Dr., Geograph, Kriesbaumen, 3157 Milken. 
Die SKUB hat diesen Zusammenhang bereits frühzeitig erkannt und auch die Bereiche Landschaft, Ökonomie und Administration ins Beobachtungskonzept eingebettet. Dies ist um so bemerkenswerter, als sich die im europäischen Raum bestehenden Beobachtungsprogramme an die traditionellen, ökologieorientierten Umweltanalysen anlehnen.

\section{Anforderungen an eine integrale Landschaftsbeobachtung}

Stellt man sich die Frage, wie man den Landschaftswandel in seinem Charakter möglichst umfassend beobachten könnte, so ist die Gefahr groß, daß man sich in der Vielfältigkeit der Landschaftsbetrachtung und der Landschaftsdefinition verliert. Landschaftsbeobachtung und Landschaftsbeschreibung ist immer einschränkend. Schon der Begriff "Integrierte Beobachtung» wirft unzählige Fragen auf. Weiter signalisieren wir mit dem Wort oder der gewünschten Tätigkeit «Beobachten» eine eingeschränkte Teilnahme am Phänomen Landschaft. Ganzheit geht aber über Beobachten hinaus. Aus der Sicht der Beobachtung bewegen wir uns unausweichlich im teilnehmenden Sinn in der Landschaft.

Ohne an dieser Stelle allzu detailliert auf das Wesen der Landschaft eintreten zu wollen, sei trotzdem der Hintergrund beleuchtet, vor welchem eine möglichst integrale Landschaftsbeobachtung durchgeführt werden könnte.

In der Kulturlandschaft erfährt der Mensch die Natur in einer praktisch-produktiven Zuwendung, die Landschaft ist für ihn die materielle Lebensgrundlage. Neben dieser ökonomischen Ausrichtung erlebt der Mensch in einer intellektuell-ästhetischen Zuwendung eine geistige und seelische Auseinandersetzung mit der ihn umgebenden Mitwelt. Die Landschaftsökologie und das Landschaftsbild sind Untersuchungsgegenstand dieser MenschLandschafts-Beziehung. Doch dieser duale Ansatz als Basis genügt nicht, um die Landschaft auch als ideellen Lebensraum zu verstehen, als Dimension der menschli- chen Psyche und des Seelenhintergrundes. Eine sich auch im Nicht-Sinnlichen (NOHL 1990) manifestierende Mensch-Natur-Beziehung beeinflußt das Landschaftserlebnis und somit auch die Landschaftsvorstellung. SCHÄRLI-CORRADINI (1992: 61) spricht vom Psychotop, ABT (1983: 120) vom Ortsgeist und RÖLLIN (1990:2) vom Raum als emotionalem Feld. Landschaft dient nicht nur zur körperlichen und geistigen Ernährung, sondern auch zur Identifikationsbildung.

Diese Sicht der Landschaft durch die Menschenbrille, ihre Reduktion auf humane Produktions-, Erlebnis- und Erfahrungsräume verführt jedoch stark zu einem Anthropozentrismus, unter welchem wir sie nur als "Landschaft für uns» wahrnehmen. In der "Landschaft an sich» eröffnet sich jedoch ein unbegreiflicher außermenschlicher Bereich, der sich der Beobachtung durch uns entzieht.

Wie wir sehen, ist die Landschaftsdiskussion keine disziplinär erschöpfbare Aufgabe, und sie darf keineswegs einer natur- oder geisteswissenschaftlich begründeten Beschränktheit unterliegen. Die Auseinandersetzung mit Landschaft muß immer auf einer Arbeitsdefinition basieren. Der Zweck der Landschaftsbeobachtung besteht in der langfristigen, integrierten Beobachtung, in der Dokumentation der Landschaftsveränderung, und es erscheint daher als zentral, den Wandel ins Blickfeld zu rücken. Über den Versuch, den Wandel dokumentieren und verstehen zu wollen, ist es möglich, sich dem Phänomen Landschaft in problemorientierter Form anzunähern.

Die Landschaftsökologie (Landschaftshaushalt), in welcher sowohl die menschliche wie auch die außermenschliche Dimension zusammenfinden (Kultur- und Naturlandschaft), das Landschaftsbild als Konkretisierung der räumlichen Realien und Fiktionen sowie die Landschafiliche Identität als Mensch-Umwelt-Beziehungsfeld sind die landschaftlich zentralen Problem- oder Arbeitsfelder im Rahmen einer integrierten Umweltbeobachtung.

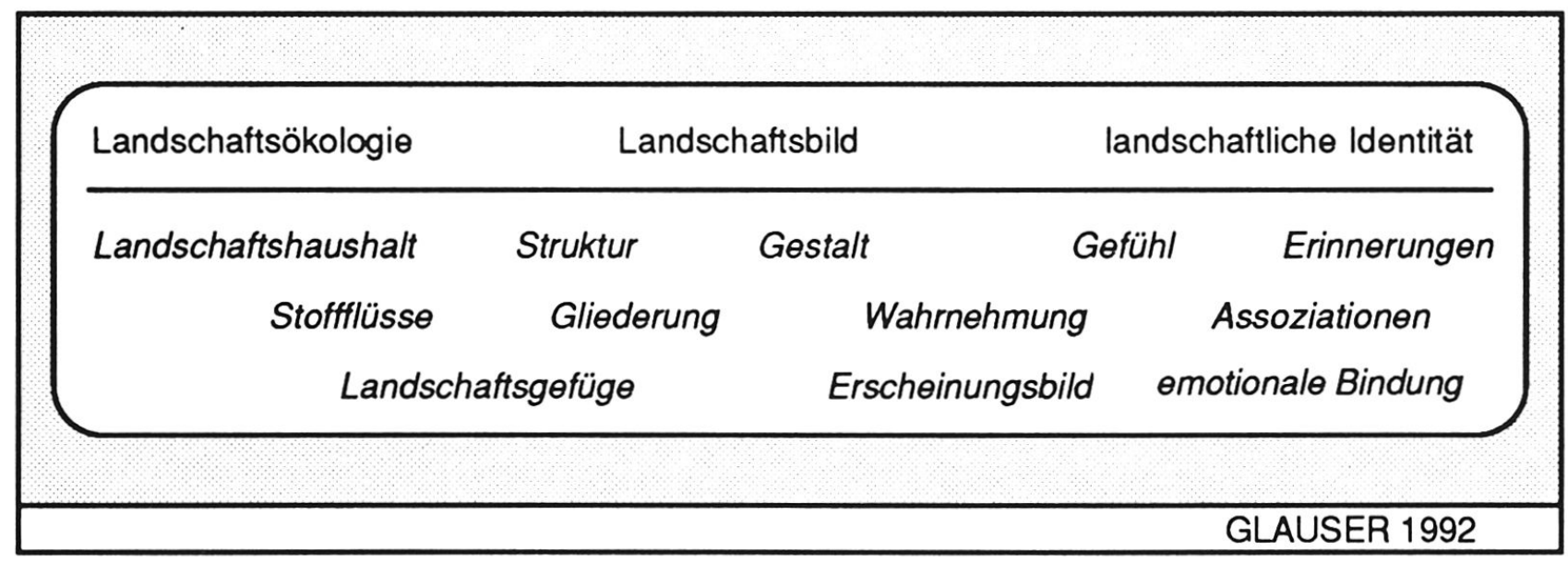

Abb.1 Landschaftliche Inhalte den Bereichen Landschaftsökologie, Landschaftsbild und Landschaftliche Identitä gegenübergestellt. 


\section{Landschaftsbeobachtung in der Schweiz, Versuch einer Übersicht und Charakterisierung}

\section{Die Beurteilungsbasis}

Wollen wir eine Übersicht und eine Beurteilung von bestehenden Ansätzen zur Landschaftsbeobachtung geben, so ist es nötig, kurz die Kriterienbasis der SKUB wiederzugeben.

a) Die bestehenden Arbeiten und Methoden sollen an den drei Bereichen Landschaftsökologie, Landschaftsbild und Landschafiliche Identität gespiegelt werden.

b) Im Rahmen der fachübergreifenden Diskussionen um die räumliche Bezogenheit von Methoden- und Parameteranwendungen wurden die räumlichen Ebenen "Objekt», "lokal», "regional» und "überregional» definiert.

c) Die Datenerhebung oder die Datenerhebungsgrundlagen müssen ein langfristiges Beobachtungsprogramm ermöglichen. Rohdaten oder klar verifizierbare und definierte Kategorien ohne großen Generalisierungsgrad bilden die beste Ausgangslage zur Langzeitbeobachtung.

d) Beobachtungsmethoden und -daten müssen ein hohes Integrationspotential aufweisen, sowohl im vertikalen (Raum) wie auch im horizontalen (Umweltbereiche) Sinn.

e) Die praktische Realisierbarkeit stellt eine wichtige Grundbedingung dar.

In der Studie zur Landschaftsbeobachtung wurden 41 Verfahren, welche sich im weitesten Sinne für die Beobachtung oder die Interpretation von Beobachtungen eignen, untersucht. Acht z. T. erweiterte Verfahren wurden im Testgebiet "Lägern" angewendet und, wo möglich, im Quervergleich besprochen. Im vorliegenden Kurzbericht wird jedoch nur auf eine generelle Charakterisierung, auf die auffallendsten Ergebnisse aus den Fallbeispielen und auf ein mögliches, dreistufiges Realisierungsprogramm hingewiesen.

\section{Generelle Übersicht}

Der fehlende prospektive Ansatz,

Sekundär- und Tertiärdaten als Grundlage

Betrachten wir die Situation der Landschaftsbeobachtung in der Schweiz im Lichte des bestehenden Datenmaterials über Kulturlandverlust und Landschaftsveränderung, so fällt auf, daß langfristige Beobachtungsreihen fehlen, ja daß ein eigentliches landschaftsspezifisches Beobachtungsmedium fehlt. Die meisten Forscher und Autoren befassen sich mit der Dynamik der Landschaft im retrospektiven Sinn, das heißt, sie sammeln Daten im nachhinein aus Quellen, die nicht speziell zur Landschaftsbeobachtung erarbeitet worden sind. Die Analyse der Landschaftsveränderung auf der Basis der Landeskartensignaturen (Methode EWALD) ist wohl das bekannteste Beispiel dazu. Solche Methoden können selten in einem breiten Bereich Aussagen zu Veränderungen von
Landschaften machen. In ganz spezifischen Bereichen weist ihre Anwendung wegen der meist problemlosen Datenbeschaffung und der bereits geleisteten Kategorienbildung jedoch große Vorteile auf (z.B. Verkehrsinfrastrukturveränderung, vgl. HÜSLER et al. 1989).

\section{Schutzspezifische Inventare}

Ein großer Teil der Methoden, mit welchen die Landschaft erfaßt wird, dienen dem Vollzug der Gesetzgebung (z. B. eidgenössische und kantonale Inventare). Meistens ist eine Eingrenzung im Beobachtungsbereich (z.B. Hochmoore) oder im Perimeter gegeben. Dabei fällt auf, daß ältere Inventare kaum auf Datenbanken basieren, sondern generell und verbal umschreiben (z. B. BLN, Baudenkmäler usw.). Die Inventarisierung hat sich jedoch über die Aggregationsstufe (Wertdaten werden aufgenommen, z. B. ISOS, kantonale Bauinventare) und über die Datenbankstufe (Datenbank wird eröffnet und als Basis zur Wertung benutzt, z. B. IVS, Moorlandschaften von besonderer Schönheit und nationaler Bedeutung) zur Proben- und Datenbankstufe weiterentwickelt (z. B in Ansätzen das Inventar der Hochmoore). Den Inventaren ist allgemein eigen, daß sie nicht zur laufenden Beobachtung ausgelegt sind und nicht periodisch wiederholt werden.

\section{Projektspezifische Datenerhebung,}

Bewertung und Aggregation

Ein ganz beträchtlicher Teil der neueren Methoden wurde zur Beurteilung von Projekten entwickelt. Es handelt sich um Inwertsetzungsmethoden, und ihnen ist eigen, daß sie meistens in bezug auf eine bestimmte Räumlichkeit und auf eine bestimmte Emissionsform entwickelt wurden. Dabei werden oft komplizierte Aggregationsverfahren angewendet, die direkt aussageorientiert sind. Attribute wie "national bedeutend", "schützenswert» oder «Risikoraum» ermöglichen höchstens die Beobachtung von veränderter Werthaltung. Diese Verfahren eignen sich kaum zur Langfristbeobachtung.

\section{Landschaftsökologische Beobachtungsansätze im Übergewicht}

Bei der Suche nach Beobachtungsmethoden fällt auf, daß der Ansatz «Beobachten» bei landschaftsökologisch ausgerichteten Methoden am meisten fortgeschritten ist. Beim Landschaftsbild, aber noch ausgeprägter bei der Landschaftlichen Identität sind beobachtungsorientierte Ansätze selten.

\section{Meistens selektive, selten repräsentative,}

kaum flächendeckende Daten

Die im Sinne der Objekte selektiven Methoden überwiegen stark. Selektive Auswahl ermöglicht eine intensivere Beobachtungstätigkeit bei größerer Detailnähe, darunter leidet jedoch vor allem der integrative Ansatz.

Repräsentative Beobachtungs- oder Aussagemethoden sind eher selten. Diese beinhalten zum einen den Vorteil, großräumig und auch politisch relevante Informationen zur Verfügung zu haben. Zum anderen leiden jedoch darunter die klare räumliche Definiertheit der Objekte 


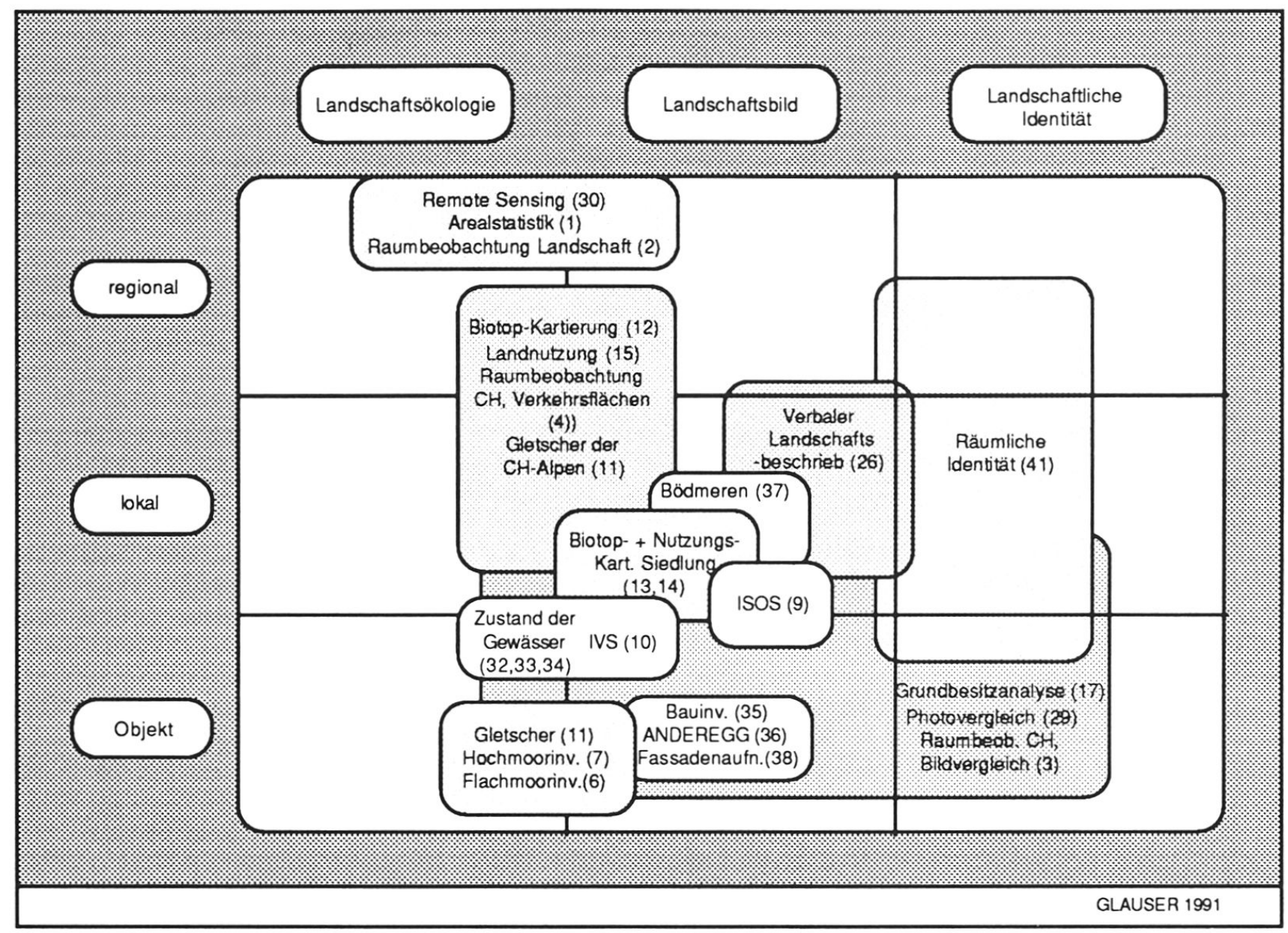

Abb. 2 Verteilung der Landschaftsbeobachtungsmethoden.

sowie die Aussagemöglichkeit zu räumlichen Interaktionsprozessen.

Nur in den wenigsten Fällen sind flächendeckende Beobachtungen mit einem klaren räumlichen Bezug anzutreffen.

\section{Auffallende Ergebnisse aus der Fallbeispieluntersuchung}

Das eigentlich erstaunlichste Ergebnis aus der genaueren Untersuchung und den Anwendungen in der Lägern ist, daß es auch bei den landesweiten Untersuchungsprogrammen zur Landschaft (Arealstatistik des Bundesamtes für Statistik, Raumbeobachtung und Amtliche Vermessung des Bundesamtes für Raumplanung, Waldbeobachtung des wSL, Bundesinventare des BUWAL usw.) keine gemeinsame Datenerhebungsstrategie besteht oder angestrebt wird. Es gibt keine Integrationsbemühungen auf der Ebene der Parameter, jedes Amt, ja jeder verantwortliche Projektleiter erfindet das Rad neu. Nachfolgend seien kurz die auffallendsten Eigenheiten beschrieben.

- Seit 1984 wird vom Bundesamt für Statistik (BFS) die Arealstatistik der Schweiz nach dem Verfahren einer stichprobenweisen Luftbildauswertung nach genau definierten Nutzungskategorien angewendet. Der Ka- tegorienkatalog ermöglicht hauptsächlich Aussagen zur Nutzung und zur Struktur. Trotz der umfangreichen Kategorienliste sind die Aussagen zu wenig differenziert. So können z. B. Wiesland und Ackerfläche nicht unterschieden werden. Demgegenüber wird zwischen "Autobahngrün» und "Flugplatzgrün» unterschieden. Weiter sind Relikte aus der ehemaligen Arealstatistik zurückgeblieben, so z. B. die Unterteilung in günstiges oder übriges Wies- und Ackerland. Solche Wertungen sind für eine langfristige Landschaftsbeobachtung nicht geeignet. Es scheint, daß der Kategorienkatalog nicht in Anlehnung an die real existierende Landnutzung entworfen wurde, sondern hauptsächlich im Hinblick auf die Auswertung von Schwarzweiss-Luftbildern im Maßstab 1:25000 des Bundesamtes für Landestopographie.

Trotz dieser Mängel ist die Arealstatistik für die Langzeitbeobachtung, im Bereich der überregionalen Quantifizierung der Bodennutzung, eines der am besten geeigneten bestehenden Informationsinstrumente. Mit dem ha-Raster ist eine Modellierung gegeben, welche mit anderen Untersuchungsmethoden gut kompatibel ist. Nach einer Bereinigung des Kategorienkataloges sind durchaus Querverbindungen zu anderen, europäischen Beobachtungskonzepten (Stati- 
stisches Informationssystem zur Bodennutzung STABIS in Deutschland, TER-UTI-Erhebung in Frankreich, CORINE Land Cover Project der EG usw.) sowie zu lokal und regional orientierten Landschaftsbeobachtungsmethoden möglich.

- Mit großem Aufwand hat das Bundesamt für Raumplanung (BRP) im Rahmen der "Raumbeobachtung Schweiz» eine Erhebung über die Landschaftsveränderungen auf der Basis von Signaturenänderungen der Landeskarte 1:25000 durchgeführt und 1992 die Resultate präsentiert. Den rund 90 Signaturen der Landeskarte werden acht Fragekomplexe zum Landschaftswandel zugeordnet. Und darin besteht bereits die erste Schwäche der Untersuchung, denn mit den vorhandenen Kartensignaturen lassen sich die gestellten Fragen eigentlich gar nicht beantworten. Die METRON (1992: 11 ) hält fest: «Vor einer Überinterpretation der Signaturenveränderung ist zu warnen. Aussagen zur qualitativen Veränderung der Landschaft können nur zu einem geringen Teil direkt, zum großen Teil indirekt erbracht werden. So können Veränderungen an Kleinstrukturen, an Waldausdehnungen und Waldrändern, Geländeformen in der Regel konsequent und aussagekräftig abgeleitet werden. Spezielle Waldstandorte, extensiv genutzte oder brachgefallene Flächen sowie das Nutzungsmuster von Land- und Forstwirtschaft dagegen werden von keinem Label erfaßt.» Eine weitere Eigenheit dieser Methode besteht darin, daß Veränderungen beobachtet werden. Der eigentliche Zustand der Landschaft wird nicht dokumentiert, und die Veränderungen stehen losgelöst von der landschaftlichen Gesamtheit da. Mit anderen Worten: Wir wissen zwar, wie viele Hecken verschwunden sind, aber nicht, wie viele noch existieren und wo sie sich befinden.

- Einen vielversprechenden Versuch stellen die Biotopund Nutzungskartierungen auf der Basis von Infrarotluftbildern ( $1: 10000)$ dar. Sowohl in der Siedlung wie im Nichtsiedlungsbereich hat das Infrarotluftbild eine zentrale Bedeutung. Basierend auf dieser multifunktionalen Probenbank und auf einem rohdatenähnlichen Parameterkatalog, gilt dieses Verfahren als integrale Ausgangslage zu Aussagen in verschiedensten Beobachtungsbereichen. Es muß an dieser Stelle jedoch festgehalten werden, daß mittels Koordination dem Wildwuchs von Kategorienkatalogen unbedingt Einhalt geboten werden muß, werden doch in der Schweiz zurzeit mehrere solcher Listen unabhängig voneinander entwickelt und angewendet (vgl. dazu GALLAND et al. [1990], CHIFFELLE [1986], BERNOWITZ \& LEUTERT [1987] oder HEGG, BEGUIN \& ZOLLER [1993]).

Dies sind nur drei Beispiele, die verdeutlichen sollen, daß die koordinierende und integrierende Hand im Bereich der Landschafts- und Umweltbeobachtung fehlt. Dies tritt um so deutlicher an den Tag, wenn wir noch die verschiedenen Bestrebungen (oder Unterlassungen) in den Kantonen mitberücksichtigen. Einer koordinierten und integrierten Umweltbeobachtung kommt, nicht zuletzt mit Blick auf die vorhandenen Mittel, eine wachsende Bedeutung zu. Der nachfolgende Vorschlag soll im Bereich Landschaft als Diskussionsgrundlage dienen.

\section{Vorschlag zu einer integrierten, langfristigen Landschaftsbeobachtung}

Die SKUB schlägt in ihrem Schlußbericht die "Ökosonde» (SKUB 1993: 78) als zu realisierenden Schritt in eine koordinierte Umweltbeobachtung vor. Ausgegangen wird von Beobachtungsgebieten im lokalen/regionalen Größenrahmen, in welchem sich die statistisch-repräsentativen Beobachtungen der heute bestehenden Meßnetze und ökosystemare Beobachtungen überschneiden sollen (SKUB 1993: 100, SCHEURER 1991: 9). Aus der Sicht einer integralen Landschaftsbeobachtung gilt es, mit geeigneten Landschaftsbeobachtungsmethoden und -verfahren Lücken im Bereich der ökosystemaren, lokal/regional ausgerichteten Beobachtung zu schließen, und zwar über den gesamten Landschaftsbereich hinweg (Ökologie, Bild, Identität). Dabei sind eine gemeinsame räumliche und zeitliche Erhebungsstrategie sowie die Koordination zu den bestehenden, geeigneten Beobachtungsverfahren prioritäre Aufgaben. Der nachfolgende Vorschlag ist diesen Vorgaben entsprechend aufgebaut. Den Realisierungsmöglichkeiten entsprechend wurde ein dreistufiges Programm entwickelt (vgl. Abbildung 3).

\section{Stufe: Dokumentationsebene (Grundprogramm)}

Den Informationsträgern oder Datenerhebungsgrundlagen kommt gerade beim Langzeitaspekt eine enorme Bedeutung zu. Im Hinblick auf zukünftige Fragestellungen, aber auch beim Berücksichtigen einer mit minimem Aufwand machbaren Umweltbeobachtung nehmen Datenerhebungsgrundlagen einen zentralen Stellenwert ein. Auch wenn aus irgendwelchen Gründen keine Angaben zu Parametern gesammelt werden können, so ist beim Bestehen einer entsprechenden Dokumentationsbasis (Probenbank) die langfristige Beobachtung, wenn auch eingeschränkt, möglich.

Als Datenerhebungsgrundlagen bieten sich an:

\section{Infrarotlufibild}

Die Stärken des Luftbildes und insbesondere des Infrarotluftbildes liegen in seiner multifunktionalen Nutzung. Das Luftbild wird von den verschiedensten umweltrelevanten Fachbereichen als Informationsträger benutzt (Klima, Biologie, Kartographie, Geomorphologie) und übernimmt bereits auf der Dokumentationsebene eine wichtige Integrationsfunktion. Zusammen mit der guten Wiederholbarkeit, der Reproduzierbarkeit und der photogrammetrischen Auswertungsmöglichkeit sind Eigenschaften gegeben, welche wichtige Voraussetzungen einer Datengrundlage sind. 


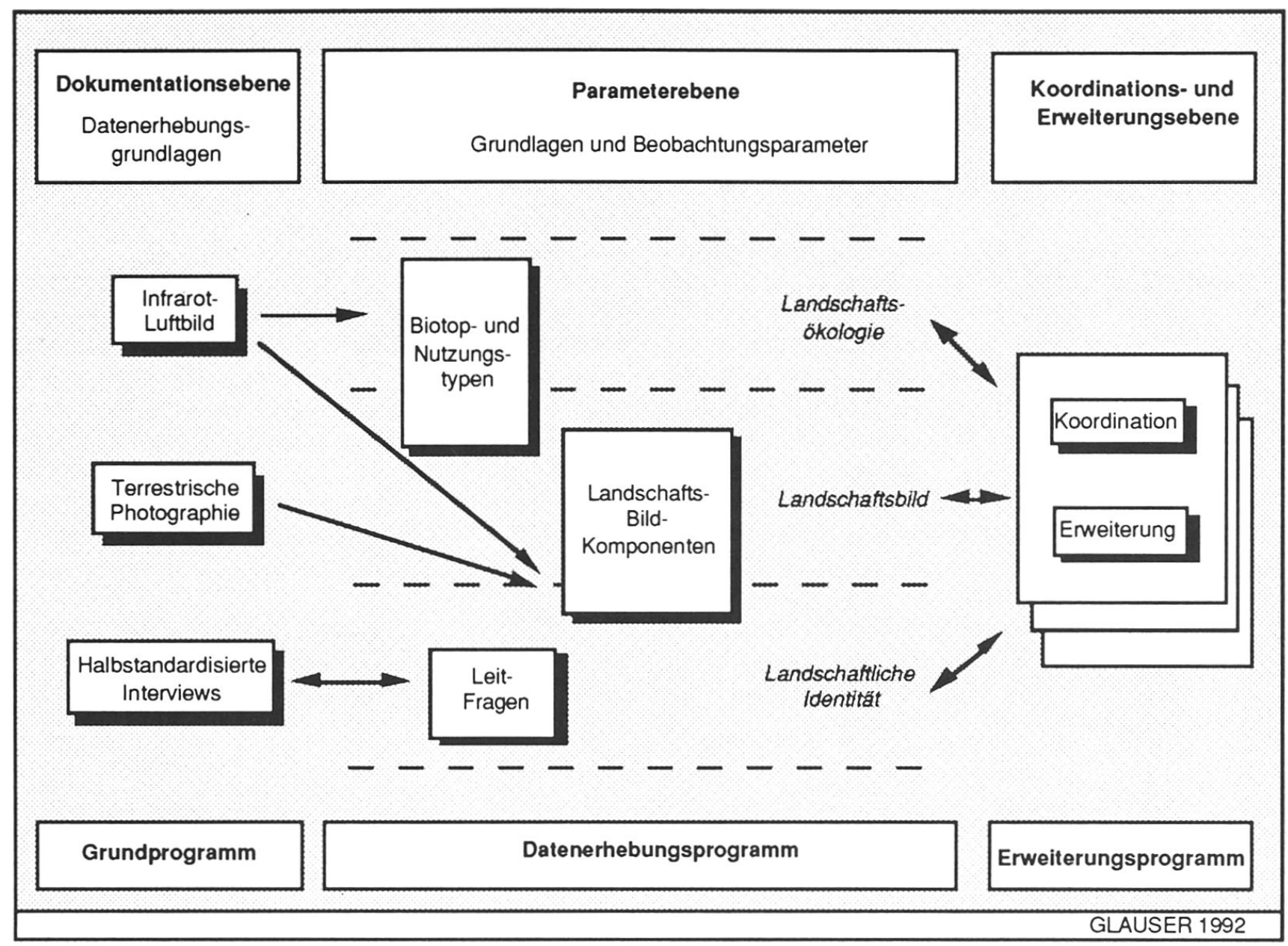

Abb. 3 Dreistufiges Realisierungsprogramm.

Terrestrische Photographie

Gerade die terrestrische Photographie hat in der Gegenüberstellung von Bildern aus unterschiedlichen Zeitepochen immer wieder sehr eindrückliche Ergebnisse erbracht. Trotzdem sind kaum systematische, kontinuierliche Beobachtungsansätze bekannt. Sicher kann gegen die Photovergleichsmethode aufgeführt werden, daß sie gar nicht flächendeckend sein kann und stark von der Standort- und Ausschnittswahl abhängig ist. Diese Einwände lassen sich jedoch auch für jede andere bildbezogene Methode vorbringen, denn Bildinterpretationen unterliegen naturgegeben stark der subjektiven Grundanordnungswahl. Gerade neuere Arbeiten zeigen, wie gut sich die Bildvergleichsmethode zur qualitativen Charakterisierung des Landschaftsbildwandels eignet und daß eine prospektive Ausrichtung diese Eigenschaft noch verbessern kann.

Die Erhebung basiert auf einem dualen Ansatz. Mit der "fixierten Gesamtansicht" sollen Panoramaaufnahmen mit Großformat- oder Laufbodenkameras gemacht werden. Mit der Wahl eines möglichst vielfältigen und repräsentativen Rundganges durch das Beobachtungsgebiet und dem Photographieren von charakteristischen Land- schaftsbildausschnitten soll die subjektive Ansicht im Objektbereich erfaßt werden (vgl. Abbildung 4).

\section{Halbstandardisierte Interviews}

Dieses Beobachtungskonzept berücksichtigt, daß die Landschaft in ihrer Bedeutung für den Menschen auch eine geistig-seelische Komponente hat. Ohne diesen Wesenszug sind Landschaftshaushalt und Landschaftsbild nicht zu verstehen und somit auch nicht integral zu beobachten.

Die Leitfragen und Intentionen bilden den Kern eines halbstandardisierten Interviews. Als Beispiel sei die zentrale landschaftsrelevante Frage von RÖLLIN (1991: 3) vorgestellt:

- "Wie lassen sich räumliche Bindungen, Fixierungen und Entfiemdungen in der aktuellen Orts- und Landschafisveränderung beschreiben? Welche Lebensräume, Landschafien, Ortstypen, Architekturen, Einrichtungen, Milieus usw: vertreten, Vertrautheit? Von welchen gehen Störungen aus?"

Rund um diese Frage lassen sich erweiterte Beobachtungsschwerpunkte aufbauen. Diese beobachtungs- 


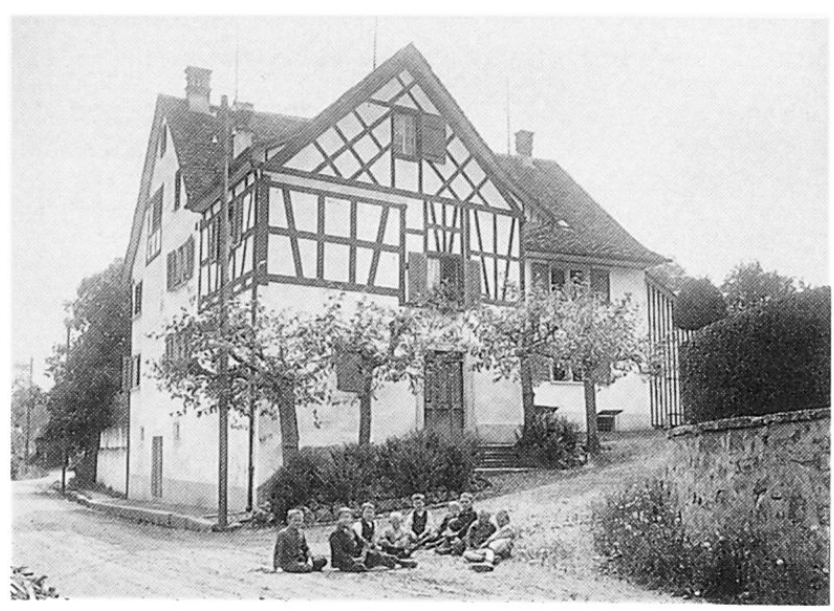

$4 \mathrm{a}$

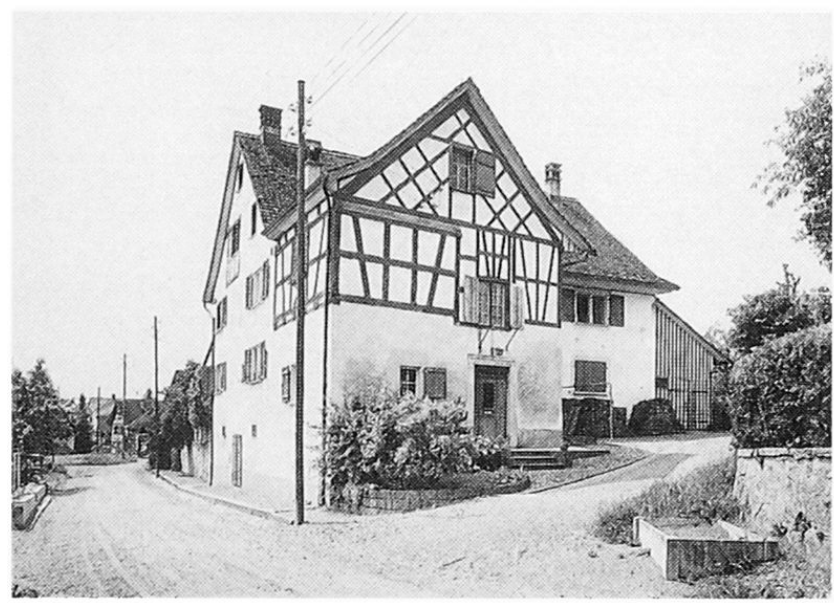

$4 \mathrm{~b}$

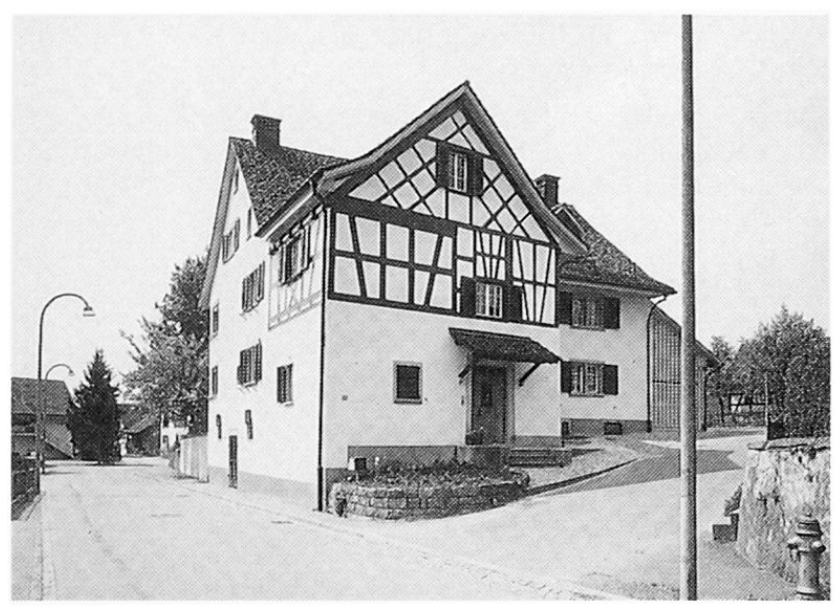

$4 \mathrm{C}$

Abb. 4 Nordostansicht des Pfarrhauses Otelfingen

a) 1908 (Photo unbekannt, Kant. Hochbauamt $\mathrm{ZH})$

b) 17.7 .1959 (Photo unbekannt, Kant. Hochbauamt $\mathrm{ZH})$

C) 8.5.1992 (Photo: Huber \& Kobi; Documenta Natura, Bern) orientierte Forschungstätigkeit im Beziehungsfeld Mensch - Landschaft wird Auskunft geben über die Operationalisierbarkeit des Ansatzes «Beobachten der Landschaftlichen Identität».

\section{Stufe: Parameterebene (Datenerhebungsprogramm)}

Klar umschriebene, reproduzierbare, rohdatenähnliche Beobachtungsparameter bilden zusammen mit den Informationsträgern/Datenerhebungsgrundlagen das Rückgrat einer Umweltbeobachtung. Für die Landschaftsbeobachtung bedeutet dies, daß auf der Basis der vorgestellten Datenerhebungsgrundlagen Parameter vorgeschlagen werden, welche in ihrer Eigenschaft die drei Bereiche Landschaftsökologie, Landschaftsbild und Landschaftliche Identität abdecken können. Drei Datenerhebungsprogramme sind von zentraler Bedeutung:

\section{Biotop- und Nutzungskartierung}

Mit der Biotop- und Nutzungskartierung steht uns eine Methode zur Verfügung, welche zum einen mit der Vegetation als dynamischem biotopischem Merkmal und zum anderen mit der kulturbezogenen Landnutzung zwei Ansätze in befruchtender Weise verbindet und geradezu prädestiniert ist, langfristige Änderungen zu dokumentieren und zu quantifizieren.

\section{Landschafisbildkomponenten}

Die eigentlichen Komponenten, nach welchen das Landschaftsbild bildbezogen festgehalten werden kann, sind eigentliche Bewertungsparameter. Gerade Bewertungsparameter unterliegen einem dauernden Werte- und Interpretationswandel. Daher muß sich die Langzeitbeobachtung auf möglichst inhärente Daten stützen können. Die direkte Abbildung der Landschaft durch die Photographie kommt dieser Forderung entgegen. Das Auswerten von Parametern ist bereits Synthesearbeit und muß immer neu erarbeitet werden. Es gibt daher keine abschließende gültige Kategorisierung.

\section{Leitfragen}

Mit dem Verfahren der halbstandardisierten Interviews ist ein Ansatz gegeben, welcher eine gute Veränderungsdarstellung der räumlichen Identität ermöglicht. Die Leitfragen, nach welchen Interviews auch immer wieder interpretiert werden können, bilden dabei eine parameterähnliche Struktur.

\section{Stufe: Koordinations- und Erweiterungsebene (Erweiterungsprogramm)}

Beim Vorschlag zu ökosystembezogenen Beobachtungsverfahren sind Koordinationsbestrebungen zu den bestehenden Beobachtungsnetzen wie der Landeshydrologie, den Moor- und Moorlandschaftsschutzinventaren, den Bauinventaren usw. vordringlich. Zudem muß auf eine Erweiterungsmöglichkeit der Datenbildung im Objektbereich Wert gelegt werden. 
Ein Verbund von repräsentativen Beobachtungsgebieten sollte zumindest konzeptionell angestrebt und organisatorisch im Auge behalten werden. «Doch es ist ratsam, alle Kräfte zunächst auf eines oder auf wenige davon (Beobachtungsgebiete) zu konzentrieren» (ELLENBERG 1991: 37). Denn nur wenn konzeptionelle Ziele auch umgesetzt werden, lassen sich konkrete Erfahrungen, aber auch überzeugende Vorschläge zu einer auf nationaler und kantonaler Ebene abgestützten, langfristigen und integralen Landschafts- bzw. Umweltbeobachtung machen.

Die Lösung unserer Landschaftsprobleme verlangt natürlich nach mehr als danach, daß sie überhaupt bemerkt werden; sie verlangt nach wirksamen Konfliktund Durchsetzungsstrategien und neuen Prioritäten. (Finke 1986: 279)

\section{Literatur}

ABT, T. (1983): Fortschritt ohne Seelenverlust. Bern: Hallwag BERNOWITZ,K., \& LEUTERT,F. (1987): Vegetationskartierung der Stadt Zürich. Zürich: Gartenbauamt der Stadt Zürich.

BURCKHARDT, L. (1985): Kinder fressen ihre Revolution. Köln: DuMont.

CHIFFELLE, F. (1986): Utilisation du sol en Suisse: In: GALLUSSER,W., et al., 1986: Siedlung, Bodennutzung und Grundeigentum der KLW-Testgemeinden in den 1970er Jahren. Studiengruppe Kulturlandschaftswandel der Schweiz, S. 56-86, Basel: Geographisches Institut der Universität.

ELLENBERG, H. (1989): Erfahrungen bei interdisziplinärer Okosystemforschung im Solling seit 1966. In: NIEVERGELT, B., \& SCHEURER, T., 1991: Forschung in Naturreservaten. Reihe: Publikationen der Schweiz. Akademie der Naturwissenschaften, Nr. 4, S. 15-40. Freiburg: Universitätsverlag.

FLACH,W. (1986): Landschaft. Die Fundamente der Landschaftsvorstellung. In: SMUDA, A. (Hrsg.), 1986: Landschaft. S.11-28. Frankfurt am Main: Suhrkamp.
GALLAND, P., et al. (1990): Typologie des milieux de Suisse. Basel/Neuenburg: Centre suisse de cartographie de la faune.

GLAUSER, P. (1993): Landschaftsbeobachtung im Rahmen einer integrierten, langfristigen Umweltbeobachtung in der Schweiz. Zürich: Geographisches Institut der Universität Zürich Irchel.

HEGG, O., BEGUIN, C., \& ZOLLER, H. (1993): Atlas schutzwürdiger Vegetationstypen der Schweiz. Bern: EDMZ.

HÜSLER, W., et al. (1989): Verkehrsflächen der Schweiz. Materialien zur Raumplanung. Bern: Bundesamt für Raumplanung.

KNOEPFEL, P. (1992): Umweltbeobachtung und Umweltberichterstattung in der Schweiz. IDHEAP: Lausanne.

METRON (1992): Landschaftsveränderung in SKUB-Testgebieten. Windisch: unveröffentlicht.

NOHL, W. (1990): Zur Rolle des Nicht-Sinnlichen in der landschaftsästhetischen Erfahrung. In: BUNDESFORSCHUNGSANSTALT FÜR NATURSCHUTZ UND LANDSCHAFTSÖKOLOGIE, 1990: Natur und Landschaft. Heft 7 /8, 65. Jg. Stuttgart: Verlag W. Kohlhammer.

PADRUTT, H. (1984): Der epochale Winter. Zürich: Diogenes. ROELLIN, P. (1991): Ortsveränderung und räumliche Identität: Kurzfassung. Nationales Forschungsprogramm 21, Kulturelle Vielfalt und nationale Identität. Basel.

SCHAERLI-CORRADINI, B. M. (1992): Bedrohter Morgen. Kind, Umwelt und Kultur. Zürich: Verlag Pro Juventute.

SCHEURER, T. (1991): Integrierte Umweltbeobachtung in der Schweiz: Erfordernisse, Ansatzpunkte und konzeptionelle Ziele. In: BEGUIN, D., et al. 1991: Zwischenbericht über die Aktivitäten des Leitungsteams und der wissenschaftlichen Mitarbeiter der SKUB. Anhang 1, unveröffentlicht.

SCHWEIZ. KOMMISSION FÜR UMWELTBEOBACHTUNG (1993): Integrierte ökosystembezogene Umweltbeobachtung; Konzept für die Einführung eines Beobachtungssystems. Ausführlicher Bericht, Bern.

WALDENFELS, B., 1986: Gänge durch die Landschaft. In: SMUDA, A. (Hrsg.), 1986: Landschaft. S. 238-265. Frankfurt am Main: Suhrkamp.

ZIMMERMANN. W. (1988): Umweltbeobachtung und Umweltforschung in der Schweiz. Dokumentation, Auswertung Schriftenreihe Umweltschutz Nr. 80 und 81. Bern: Bundesamt für Umweltschutz. 